\title{
A Topological Magueijo-Smolin Varying Speed of Light Theory, the Accelerated Cosmic Expansion and the Dark Energy of Pure Gravity
}

\author{
Mohamed Salah El Naschie1, Leila Marek-Crnjac², Mohamed Atef Helal'3 , Ji-Huan He \\ ${ }^{1}$ Department of Physics, Faculty of Science, University of Alexandria, Alexandria, Egypt \\ ${ }^{2}$ Technical School Center of Maribor, Maribor, Slovenia \\ ${ }^{3}$ Department of Mathematics, Faculty of Science, Cairo University, Cairo, Egypt \\ ${ }^{4}$ Nantong Textile Institute, National Engineering Laboratory for Modern Silk, College of Textile and Clothing, \\ Soochow University, Suzhou, China \\ Email: Chaossf@aol.com, ${ }^{*}$ leila.marek@guest.arnes.si, mahelal@yahoo.com, hejihuan@suda.edu.cn
}

Received 10 March 2014; revised 15 April 2014; accepted 23 April 2014

Copyright (C 2014 by authors and Scientific Research Publishing Inc.

This work is licensed under the Creative Commons Attribution International License (CC BY).

http://creativecommons.org/licenses/by/4.0/

(c) (i) Open Access

\section{Abstract}

The paper presents a detailed analysis of ordinary and dark energy density of the cosmos based on two different but complimentary theories. First, and starting from the concept of the speed of light being an average over multi-fractals, we use Magueijo-Smolin's ingenious revision of Einstein's special relativity famous formula $E=m c^{2}$ to a doubly special formula which includes the Planck energy as invariant to derive the ordinary energy density $E(O)=m c^{2} / 22$ and the dark energy density $E(D)=m c^{2}(21 / 22)$ where $m$ is the mass and $c$ is the speed of light. Second we use the topological theory of pure gravity to reach the same result thus confirming the correctness of the theory of varying speed of light as well as the COBE, WMAP and Type 1a supernova cosmological measurements.

\section{Keywords}

Dark Energy, Quantum Gravity, Varying Speed of Light Theory, Energy of Quantum Wave, Quantum Non-Demolition

\footnotetext{
"Corresponding author.
}

How to cite this paper: El Naschie, M.S., Marek-Crnjac, L., Helal, M.A. and He, J.-H. (2014) A Topological Magueijo-Smolin Varying Speed of Light Theory, the Accelerated Cosmic Expansion and the Dark Energy of Pure Gravity. Applied Mathematics, 5, 1780-1790. http://dx.doi.org/10.4236/am.2014.512171 


\section{Introduction}

The familiar speed of light is the well-known constant value only in the vacuum. The speed of light is otherwise dependent upon the density of the medium which the light passes through. The situation with a fractal spacetime is not dissimilar. Imagining a fractal space-time to be infinitely many layers, each with a different number of dimensions depending upon the resolution of observation, we could regard the speed of light as variable but with a constant expectation value taken as the statistical averaging over infinitely many speeds of slight ranging from infinitely small to infinitely large. Looking at things in this way we will apply the idea of varying speed of light to obtain an accurate estimation for the missing dark energy and the surprising acceleration rather than deceleration of cosmic expansion.

In the present paper we use Magueijo-Smolin’s varying speed of light (VSL) theoretical modification of Einstein's energy mass relation to derive an exact value for the missing dark energy which is found to be in astonishing agreement with the latest result of the WMAP measurement and the independent super nova analysis. Thus while Einstein's formula predicts 95.5\% more energy than found in highly precise astrophysical measurement, our VSL based calculation indicates an exact theoretical value of only $4.508497 \%$ real energy. Consequently the exact conjectured missing dark energy must be $95.491502 \%$. This is a clear cut confirmation for both the cosmological measurement and the VSL theory. However, this is far from being the final resolution of the riddle because the missing dark energy of the cosmos has a profound real effect reflected in the observed increased rather than decreased rate of the cosmic expansion of the universe mentioned earlier on. It then turned out that the dark energy is the 95.5\% energy of the quantum wave which is a Legendre transformation in dual complementarity to the ordinary $4.5 \%$ energy of the quantum particle. On the other hand measurement leads to quantum wave collapse preventing the detection of its propagation energy. Thus we are left with a single rational conclusion, namely that quantum non-demolition measurement instruments must be developed first if we are to have a chance in finding the missing 95.5\% dark energy. We stress that the ability of the present theory to make such accurate predictions is uniquely tied up with incorporating into it, the measure zero Cantor set behind Hardy's quantum entanglement and the associated empty set of the vacuum or pure gravity of the general theory of relativity.

\section{Background Information and Agenda}

The issue of missing dark matter and worse still missing dark energy in the cosmos is since some time now a colossal problem threatening the very foundations of theoretical physics and cosmology [1] [2]. There is in the meantime objectively undeniable confirmation via the sophisticated analysis of COBE, WMAP and super nova measurement that approximately $95.5 \%$ of the energy which the cosmos should in theory contain is not there to see or measure in any direct way [1].

In the present work we give an exact analysis based on a topological theory of varying speed of light pioneered by J. Magueijo and L. Smolin showing that the maximal energy density value predicted by Einstein's theory of relativity $E=m c^{2}$ where $E$ is the energy, $m$ is the rest mass and $c$ is the velocity of light gives the wrong answer when it comes to calculating the intergalactic energy scales [1] [2]. The correct equation in this case must take quantum mechanics or equivalently hyperbolic non-classical fractal topology of the fabric of space-time and the vacuum, i.e. the pure gravity of general relativity into account [2]-[8] in order to bring theoretical predictions and experimental measurement together [8]-[25]. To this end we will use a topological variant of the equation developed by Magueijo and Smolin [9] [10] to modify Einstein's relativity theory using the theory of varying speeds of light (VSL). Subsequently, we give the VSL equation a novel topological interpretation using an eleven dimensional theory akin to that of super gravity and M-theory but within a fractal setting [7] [8]. At the end the so-obtained result confirms the non-classical nature of $95.4915 \%$ of the energy of the cosmos as well as the experimental findings based on the COBE, WMAP, Planck and super nova measurement which indicates 95.5\% dark energy [1] [2]. The amazing agreement between theory and experiment is unparalleled and leaves very little room for doubting the cosmological measurement [1] or the VSL theory [9] [10] nor indeed for that matter, the fractal Cantorian nature of space-time at the quantum scales [3]-[8]. Never the less this is by no means the end of the story. In the first instance the problem of the missing dark energy seems to be resolved by finding a missing $1 / 22$ factor in $E=m c^{2}$ which should be $E$ (Ordinary) $\cong m c^{2} / 22$. However, where is $E$ (Dark) $\cong$ $m c^{2} /(21 / 22)$ which together with $E$ (Ordinary) gives $E$ (Einstein)? This is still a more than valid and meaningful 
question because this dark energy has a real profound effect reflected in the measured increase rather than decrease of the rate of expansion of the cosmos [1]. The answer to this riddle turned out to be in the nature of the degrees of freedom of the pure energy vacuum of Einstein's field equations as well as the wave-particle duality and the quantum measurement problem which are two fundamental aspects of orthodox quantum mechanics [21]-[25]. Applied to ordinary energy and dark energy we found that the $4.5 \%$ is the energy of the quantum particle as modeled by the zero set in five dimensional Kaluza-Klein space-time while the $95.5 \%$ is the dark energy of the quantum wave as modeled by the empty set in the same $\mathrm{D}=5$ space-time (see Figure 1(A) and Figure 1(B)) [23]-[25]. The same result could be obtained however even more directly by looking carefully at the degrees of freedom of pure gravity in the absence of any matter field. On the other hand we know very well that measurement causes a state vector reduction i.e. the propagation energy of the quantum wave could not possibly be measured unless new sophisticated quantum non-demolition measurement instruments are developed [22] which at present are out of reach if at all possible [21].

The present analysis is structured logically as follows:

1) After the introduction and the background information we give the quintessence of the VSL theory in Section 3.

2) In Section 4 we show the consistency of the Magueijo-Smolin formula with Newtonian mechanics and Einstein's relativity, then derive the ordinary energy density and the dark energy density.

3) In Section 5 the importance of quantum entanglement interpretation of the obtained results is explained in the light of Hardy's celebrated formula for quantum entanglement of two quantum particles.

4) Finally the set theoretical interpretation of ordinary energy and dark energy is explained in Section 6 while Section 7 wraps it all in terms of the topological theory of pure gravity.

\section{The Energy-Mass Relation of the VSL Theory}

The completely new idea behind the derivation of the energy-mass relationship using the VSL theory basically comes from H. Weyl inspired theory of scale relativity [3]. Both scale relativity and VSL theory [9] [10] take the Planck energy

$$
E_{p}=1 / l_{p}
$$

where $l_{p}$ is the Planck length as invariant. Consequently J. Magueijo and L. Smolin arrive at their replacement of Einstein's single equation $E=m c^{2}$ by three remarkable and thoroughly ingenious equations [9]

$$
E=\frac{m_{0} c^{2}}{1+\frac{m_{0} c^{2}}{E_{p}}}
$$

with the usual $m$ transformation

$$
m=\gamma m_{0}
$$

where $\gamma$ is the Lorentz factor but with $E$ and momentum $P$ transforming as [9]

$$
E=\frac{m}{1+\frac{m}{E_{p}}}
$$

and

$$
P=\frac{m v}{1+\frac{m}{E_{p}}}
$$

The preceding equations give us the possibility of admitting a ratio $m_{0} c^{2} / E_{p}$ far larger than unity. In other words through the postulates of VSL theory $m_{0} c^{2}$ can become many times larger than $E_{p}$ without violating Lorentzian invariance, nor the $1 / l_{p}$ invariance of the theory [9] (for summary and comparison with other theories see Table 1 and comment there in). To show that this superficially contradictory statement leads to a true formula for quantum relativity and gives results in full agreement with the cosmological measurements is our next and main task [24]. 
(A) The set theoretical particle-wave duality [23] [24] and comparison to pure gravity:

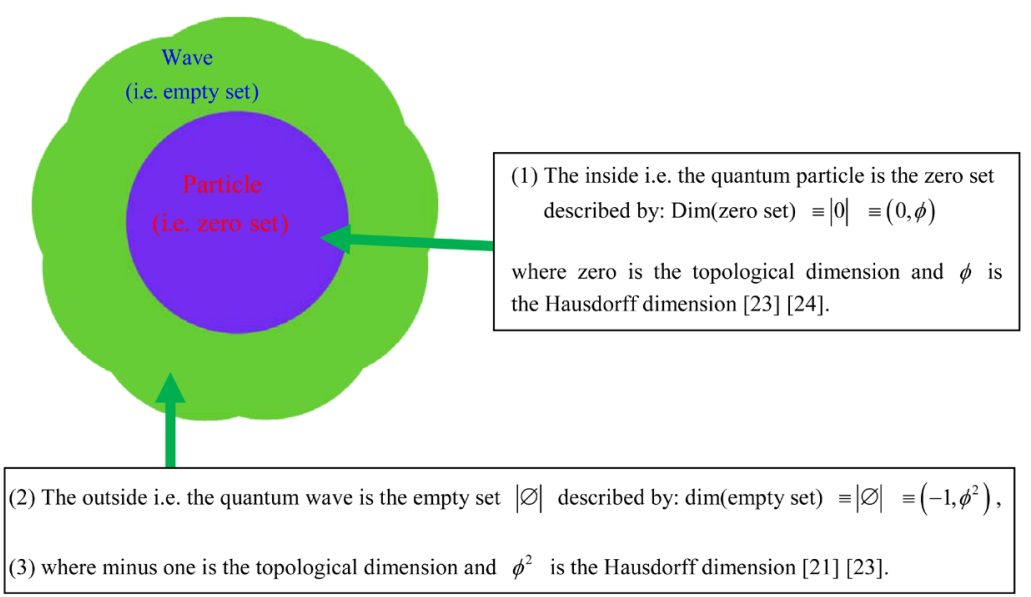

Conclusion: The quantum wave is the cobordism of the quantum particle i.e. its surfaces [23] or more poetically its halo. Measurement interferes with the empty set and renders it a non-empty zero set. Consequently the quantum wave is reduced to a quantum particle. For pure gravity given by $D=d(d-3) / 2$ the zero set corresponds to $d=3$ and $D=0$ while the empty set is given by $d=2$ and $D=-1$.

(B) Lifting the zero set quantum particle $|0|$ and the empty set quantum wave $|\varnothing|$ to five dimensional Kaluza-Klein space-time [13] [24] [25] one finds a contra part to the particle-wave duality, namely ordinary energy-dark energy duality:

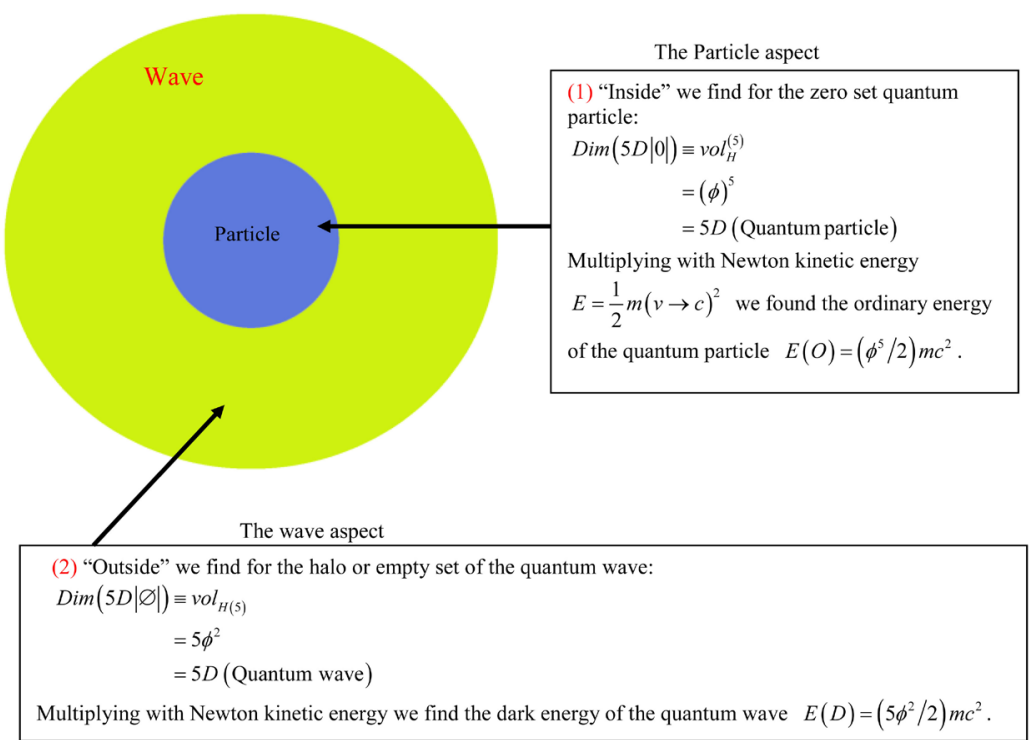

Measurement and the cause of dark energy

Measurement causes the empty set to become a non-empty zero set and consequently the quantum wave becomes a quantum particle. That is the simplest rational mathematical explanation for the wave collapse or quantum jump which puzzled people like Einstein and Schrödinger [5] [21]. On the other hand wave collapse change the $95.5 \%$ dark energy of the quantum wave to a mere $4.5 \%$ ordinary energy of the quantum particle. That is why we cannot detect nor utilize the dark energy of the propagating quantum wave using our present measurement instrument [22].

Figure 1. (A) Ordinary Energy, Dark Energy and Einstein’s Energy from the view point of set theory and quantum wave collapse as well as pure gravity in the absence of matter field associated with Einstein's field equation degrees of freedom $D=d(d-3) / 2$ where $d$ is the dimension. The contra part of the zero set quantum particle in this case is given by $d=3$ and $D=0$ while the contra part of the quantum wave empty set is given by $d=2$ and $D=-1$; (B) Quantum Measurement and dark energy of the quantum wave. 
Table 1. Explicit correspondence transforming Joao Magueijo-Lee Smolin, El Naschie and Einstein formulas into each other.

\begin{tabular}{ccc}
\hline Joao Magueijo-Lee Smolin & El Naschie & Einstein \\
Conventional $m c^{2} \rightarrow m c^{2}$ & $m c^{2}$ & $m c^{2}$ \\
Topological $m \rightarrow 5$ & & \\
Topological $c \rightarrow \phi=(\sqrt{5}-1) / 2$ & $\gamma=\left(\phi^{5} / 2\right)=\frac{1}{22+k} \simeq \frac{1}{22}$ & $\gamma=1$ \\
Topological $\quad E_{p} \rightarrow \phi^{5}$ & & \\
$\gamma \rightarrow \frac{1}{1+(5)(1 / \phi)^{3}}=\frac{1}{22+k} \simeq \frac{1}{22}$ & $E=\left(\phi^{5} / 2\right) m c^{2} \simeq \frac{m c^{2}}{22}$ & $E=m c^{2}$ \\
Final Result $E=\frac{m c^{2}}{1+(21+k)} \simeq \frac{m c^{2}}{22}$ &
\end{tabular}

There is an entire web of relations in the above constituting a complete topological "physical” dimensionless theory behind the conventional facade. For instance the dimensionless topological Plank energy $E_{p}=P(H)=\phi^{5}$ means that the topological Planck length corresponding to $10^{33}$ centimeters must be: $l_{p}=1 / E_{p}=1 / \phi^{5}=11+\phi^{5}$. This is identical to the Witten-He fractal version of M-Theory. Similarly Sigallotti's critical value and Taiji speed of light might be usefully interpreted as a topological unit interval light velocity $c=\phi$ while Newton's topological gravity constant is given by $G_{N}($ topological $)=\left(11+\phi^{5}\right)^{2}=122.9918689$. Note that $(11)^{2}=121$ and that $\sqrt{\frac{|E 8 E 8|-12}{4}}=\sqrt{121}=11$ while $\sqrt{\frac{\left(496-k^{2}\right)-4}{4}}=\sqrt{122.9918694}=11+\phi^{5}$.

\section{Dark Energy for the Extra Dark Dimensions}

From the first equation of Magueijo-Smolin [9] [10] it is clear that our crucial ratio $m_{0} c^{2} / E_{p}$ could take three vital values:

a) $m_{0} c^{2} / E_{p} \cong 0$

i.e. we have Einstein's classical equation of special relativity.

b) $m_{0} c^{2} / E_{p}=1$

i.e. we have the Newtonian expression for energy when setting $c=v$ and find $E=(1 / 2) m v^{2}$.

c) $m_{0} c^{2} / E_{p}=21$.

This is a crucial situation for us as we will explain shortly. The energy in this case could be written as

$$
E=\frac{m_{0} c^{2}}{1+21}=\frac{m_{0} c^{2}}{22} .
$$

Let us consider the above from the viewpoint of space-time dimensions [7] [8] and in particular from the view point of the theories involving extra space time and compactified dimensions as summarized in Table 2 [7]-[18].

In case (a) above we could say that space and time fuse into $3+1=4$ dimensions. There are zero hidden dimensions in this theory. The total energy $E$ is not reduced or diluted by extra dimensions.

In case (b) above we could say, somewhat surprisingly, that we have a hidden dimension, namely time appearing only as a parameter. The energy $m c^{2} \rightarrow m v^{2}$ is divided therefore by 2 .

On the other hand in the most important case concerning the present work i.e. case (c), we could interpret the factor 21 as the number of dark dimensions of 26 bosonic string theory [7] [8] [17] when a 5 dimensional Kaluza-Klein theory is used. Alternatively we take $1+21=22$ factor to mean the 22 compactified [17] invisible, dark and hidden dimensions of non-super symmetric string theory [16] [17]. In fact the 22 dark dimensions could be interpreted differently as 11 bosons and 11 fermions of a super symmetric 11 dimensional M-theory [11]-[13]. That way we have a new modified energy-mass relation almost identical to Einstein's equation with the exception that the energy is now reduced by a factor of $1 / 22$. This reduction amounts to diluting or spreading thinly Einstein's energy because of the extra dimensions which exist but were not taken into account in the derivation of the 4D theory of Einstein [24] [25]. 
Table 2. Bosonic strings interpretation of $E_{Q R}=m c^{2} / 22$ and varying speed of light theory [9] [10] $E=\frac{m c^{2}}{1+\frac{m c^{2}}{E_{p}}}$.
$\begin{gathered}\begin{array}{c}\text { Starting from Einstein's 4D space-time and } \\ \text { the 26D of Bosonic strings we have }\end{array} \\ \begin{array}{c}\text { Starting from Kaluza-Klein 5-D space-time and } \\ \text { the 26D of Bosonic strings we have }\end{array} \\ E_{Q R}=\frac{E(\text { Einstein })}{D^{(26)}-D^{(4)}}=\frac{m c^{2}}{D^{(26)}-D^{(4)}}=\frac{m c^{2}}{26-4}=m c^{2} / 22\end{gathered} \quad E=\frac{E(\text { Einstein })}{1+\frac{m c^{2}}{E_{P}}}=\frac{m c^{2}}{1+\left(D^{(26)}-D^{(5)}\right)}=\frac{m c^{2}}{1+(26-5)}=\frac{m c^{2}}{1+21}=m c^{2} / 22$

Note that: $E_{p}$ is the Planck energy $E_{p}=\left(h c^{5} / G\right)^{1 / 2}$. Setting $h / G=\phi^{5}$ and $c$ equal to Sigalotti's critical value $c=\phi=(\sqrt{5}-1) / 2$ we find that $E_{p}=\phi^{5}$ and that the rectangular area $E_{p}=\phi^{2} \phi^{3}=\phi^{5}$ is Hardy's probability of quantum entanglement while the triangular area $(1 / 2) \phi^{2} \phi^{3}=(1 / 2) \phi^{5}$ is the $\gamma$ factor in $E_{Q R}=\phi^{5} / 2 m c^{2} \cong m c^{2} / 22$. Here $\phi^{2}$ is the local entanglement and $\phi^{3}$ is the global (counter factual) entanglement of Hardy's quantum entanglement [5].

Therefore we can write [9] [26]

$$
E_{\max }=(1 / 22) m_{0} c^{2} \cong(0.04545) m_{0} c^{2} .
$$

This result is tantamount to saying that $(1-0.04545)(100)=95.454 \%$ of the energy in the universe is dark energy. The result is very close indeed to the experimental measurement of 95.5\% [1] and means that the extra dark extra dimension of micro space-time is the cause for dispersing energy to the extent that we notice only a fraction equal to $4.5 \%$ of the classically predicted total. On the other hand we know that fractal space-time implies extra dark dimensions as well as non-classical microscopic mechanics which we perceive as quantum mechanics [4]-[6]. Thus we will look once more at the preceding result from the viewpoint of quantum mechanics and thus quantum relativity, i.e. quantum gravity [3]-[5] [13] [15]-[17].

\section{Hardy's Quantum Entanglement behind Dark Energy}

The classical Heterotic string theory [17] stipulates three dimensions, namely 26, 16 and $26-16=10$ superstring dimensions of which 6 are compactified to give a 4 dimensional classical or relativistic space-time [18]. Setting this theory in fractal dressing one finds $26+k, 16+k$ and $26+k-(16+k)=10$ where $k=0.18033989$. Thus our hidden dark dimensions of the previous analysis are made "fractally" accurate by writing

$$
26+k-4=22+k
$$

where $k=\phi^{3}\left(1-\phi^{3}\right)=0.18033989$ and $\phi=(\sqrt{5}-1) / 2=0.618033989$. The exact energy $E_{\max }$ is consequently [23]-[27]

$$
E_{\max }=\left(\frac{1}{22+k}\right)\left(m_{0} c^{2}\right)=\frac{1}{2}\left(\phi^{5}\right)\left(m_{0} c^{2}\right)
$$

The remarkable thing is that $\phi^{5}$ is the exact theoretical value which Hardy found for a generic quantum entanglement of two quantum particles [19]. This value was also experimentally confirmed with high precision and sophisticated quantum informational methods using modern beam splitters etc. [5] [6] [19] [20].

In addition, the same previous result can be found directly from a delicate light cone quantized Lorentzian transformation [18] and confirms the intuitive initial hunch that $E=(1 / 2)\left(\phi^{5}\right)\left(m_{0} c^{2}\right)$ is a quantum gravity formula for quantum relativity. Consequently we could rephrase our hidden dark dimensions interpretation [7] [14] [15] to mean that the inclusion of quantum entanglement $\phi^{5}$ of a single particle i.e. $\phi^{5} / 2$ is clearly the cause for the $95.5 \%$ reduction in the classical energy prediction and therefore gives a quantum gravity explanation for the missing dark energy [12] [24]. There are numerous other interpretations of which we note the world sheet interpretation. This is based on the fact that $5 \phi^{2}+\phi^{5}=2$, i.e. the dimension of the string world sheet and consequently $\phi^{5} / 2$ and $5 \phi^{2} / 2$ are relative density. We also note the duality of the ratio of the 3D topological mass $m=\phi^{3}$ and the 5D topological mass $m=5$. Setting $5 / \phi^{3}$ in $E$ leads to the ordinary energy density via equation 1 . Dark energy on the other hand is obtained from $\phi^{5} / 5$ [23]-[27]. 


\section{The Ordinary Energy of the Quantum Particle and the Dark Energy of the Quantum Wave}

Set theoretical reformulation of orthodox quantum mechanics leads to some truly insightful realization concerning wave collapse, measurement and the fractal-Cantorian non-classical nature of the geometry of the fabric of space-time [23]. Within this mathematical formalism the quantum particle is described by a bi-dimensional zero set $(0 ; \phi)$ where the zero is the topological dimension of the particle while $\phi=2 /(1+\sqrt{5})$ is the Hausdorff dimension of the same particle. The quantum wave on the other hand is described by the empty set $\left(-1 ; \phi^{2}\right)$ which is the cobordism of $(0 ; \phi)$ describing the particle [23]. Since any attempt for measurement will cause an empty set to change into a non-empty zero set, quantum measurement is no more a mystery but a trivial fact which follows from the basic principles of set theory and the Menger-Urysohn theory of dimensions. It turns out that while ordinary energy is a multiplication of Newton-Einstein kinetic energy $E=(1 / 2) m(v \rightarrow c)^{2}$ with a five dimensional zero set $\left(\phi^{5}\right)$ which represents Hardy's quantum entanglement and leads to $E_{Q R}=\left(\phi^{5}\right)(1 / 2) m c^{2}$ (see Figure 1(B) and Figure 2), dark energy is given by the same kinetic energy but this time multiplied by the five dimensional empty set $\left(5 \phi^{2}\right)$ leading to $E(D)=\left(5 \phi^{2} / 2\right) m c^{2}$ (see Figure 3 ). Two profound results follow from the above [23]-[25]:

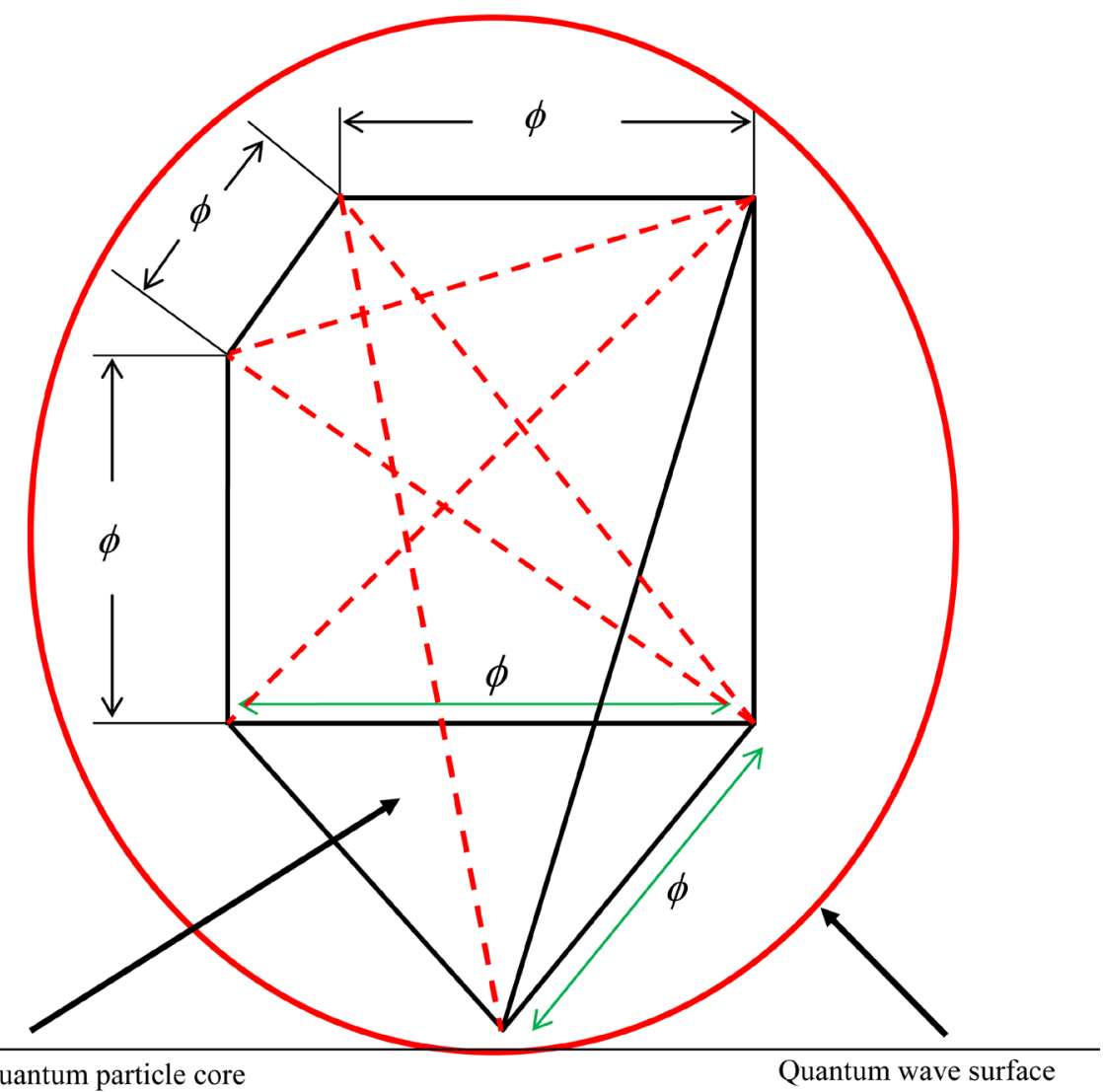

\footnotetext{
Quantum particle core

Quantum wave surface

The projection of a naive visualization of the volume $\phi^{5}$ of a five dimensional cube which decides upon the magnitude of ordinary energy of the quantum particle where $\phi=2 /(1+\sqrt{5})$.

Note that the set theoretical operation corresponding to $\phi^{5}$ is the intersection of sets, while in probability theory it is the multiplication theorem. We stress once more the fact that in 5 dimensions the volume of the sphere is a maximum larger than any other sphere in smaller or larger dimensions. That is why we use $D=5$ Kaluza- Klein space-time [13]. Here $\operatorname{Vol}\left(D_{0}\right)$ is the volume of the zero set in $D=5$.
}

Figure 2. Naïve geometrical interpretation of $\operatorname{Vol}\left(\mathrm{D}_{0}\right)=\phi^{5}$ of the ordinary energy of the quantum particle $E$ (Ordinary) $=\left(\phi^{5} / 2\right) m c^{2}$. 


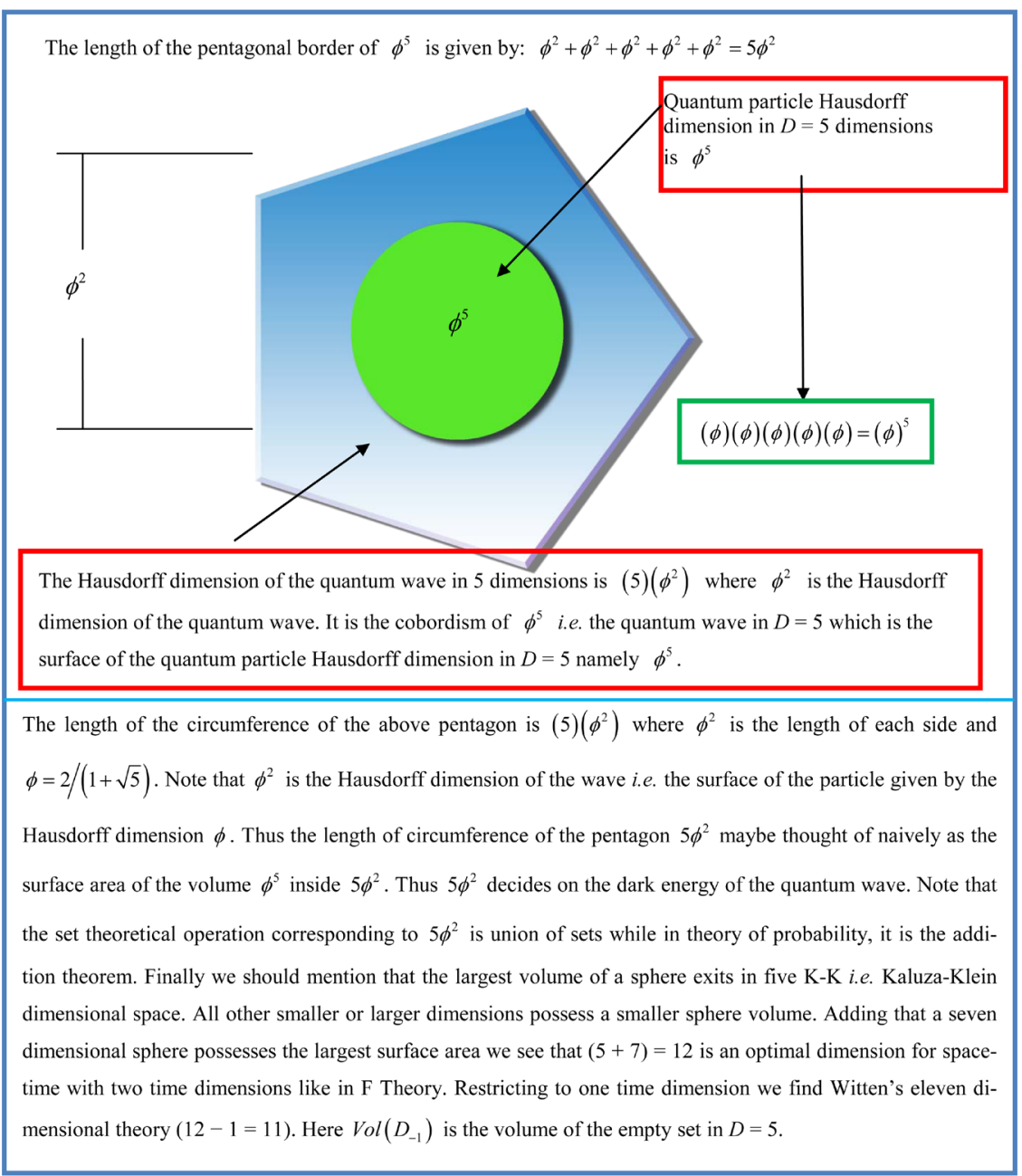

Figure 3. Naïve geometrical interpretation of $\operatorname{Vol}\left(D_{-1}\right)=5 \phi^{2}$ which decides upon the magnitude of dark energy of the quantum wave [24]-[27] $E($ Dark $)=\left(5 \phi^{2} / 2\right) m c^{2}$.

The first result is that Einstein's formula $E=m c^{2}$ is correct but totally blind to any distinction between the ordinary energy $E(O)=\left(\phi^{5} / 2\right) m c^{2}$ which we can measure and amounts to $4.5 \%$ and the dark energy $E(D)=\left(5 \phi^{2} / 2\right) m c^{2}$ which we cannot measure because of wave collapse [21] and which amounts to $95.5 \%$.

Added together one finds a new confirmation of Einstein's equation without its limitation, namely [23]-[25]

$$
E(\text { Total })=E(O)+E(D)=\left|\left(\phi^{5} / 2\right)+\left(5 \phi^{2} / 2\right)\right|\left(m c^{2}\right)=E(\text { Einstein })
$$

Here $E(O)$ plays the role of potential energy while $E(D)$ plays the role of kinetic energy.

The second result is that the only hope to measure dark energy is to develop new non-demolition measurement instruments [21] [22] something which may open new vistas in atomic reactors technology bordering on the demarcation line separating science fact from science fiction.

\section{Pure Gravity and Dark Energy from Einstein's Vacuum of His Field Equations}

When the present Authors obtained the ordinary energy density $E=m c^{2} / 22$ they were quite mindful of the fact that this unexpected fundamental result is coming primarily from the special theory of relativity rather than Einstein's general theory of gravity. In retrospect it is now clear why. Special relativity does not depend in any fundamental way or include a matter field, only 4D space-time is all what we start and end with. By contrast we tend generally to think of Einstein's field equation in the absence of any matter field as trivial. However, many 
deep thinkers of theoretical physics were intrigued by the fact that pure gravity is governed by various differential equations arising from general relativity with remarkable consequences. It is one of the most important findings of the present work that we can give a physical interpretation of the zero set of the quantum particle and the empty set of the quantum wave via the degrees of freedom of pure gravity $\mathrm{D}$ in dimension $d=3$ and $d=2$. The fundamental equation governing this behavior is the same equation for the Vierbein as well as that of the massless graviton, namely

$$
D^{(d)}=d(d-3) / 2 \text {. }
$$

for $d=3$ corresponding to a classical particle $D=0$ converting it to a de facto quantum particle being deprived of local degrees of freedom. The corresponding Hausdorff dimension is thus $\phi$ and the analogy to the zero set becomes obvious. For $d=2$ on the other hand $D=-1$ which means that we have a negative degree of freedom. Such a physically outrageous result is actually a blessing in disguise because it is saying loudly that degrees of freedom and dimensions in this case are practically the same thing and consequently we are dealing with an empty set, ergo a quantum wave which in five dimensions gives us our expected result, namely

$E(D)=\left(5 \phi^{2} / 2\right) m c^{2} \cong m c^{2}(21 / 22)$. In addition we could look upon $\gamma \cong 1 / 22$ as a running coupling constant in a similar way of how we estimate for instance the non-super symmetric quantum gravity coupling as the ratio of $\operatorname{dim} E 8 E 8=496$ to the degrees of freedom of the standard model, i.e. 12 and finding correctly that $\alpha_{g} \cong 12 / 496 \cong 1 / 42$.

In the present case we find first $D=4(4-3) / 2=2$ for $d=4$, which represents classical space-time, then we find $D$ for $d=11$ of Witten's M-theory which comes to $D=11(11-3) / 2=44$. Our $\gamma(O)$ is thus given by $\gamma(O)=2 / 44=1 / 22$ and consequently $E(O)=m c^{2} / 22$. The Legendre transformation $\gamma=1-\gamma(O)=21 / 22$ is then recognized as leading to a complimentary energy which is nothing but our dark energy with negative pressure $E(D)=m c^{2}(21 / 22)$ induced by the hyperbolic fractal geometry of a Rindler space-time and the associated anticlastic negative curvature as discussed in earlier contributions [26].

\section{Conclusions}

We give a topological interpretation and a hidden dark dimension interpretation to the Magueijo-Smolin energy mass equation of VSL theory [9] [10]. The quantitative result fully explains the missing dark energy of the cosmos [1] [2]. Einstein's equation predicts $E=m c^{2}$. However this is a total maximal energy representing the $100 \%$ theoretical density. The VSL equation on the other hand predicts the measurable ordinary energy which is approximately $1 / 22$ of this value. Accurate calculation shows that $22=26-4$ hidden dark dimensions of bosonic string theory should be 22.18033989 when taking the "fine structures" of the strings into account and consequently the exact reduction is not $1 / 22$ but

$$
1 /(22+k)=\phi^{5} / 2
$$

where $\phi=2 /(1+\sqrt{5})$. On the other hand Hardy's quantum generic entanglement for two particles is given exactly by $\phi^{5}$, a quantity which was found experimentally [5] [6] [19]. Consequently the reduction factor in the energy which is the cause for suspecting a $95.5 \%$ missing dark energy in the cosmos is clearly due to the inclusion of the quantum entanglement effect of a single particle in Einstein's formula to elevate it from a relativistic formula to a quantum relativity formula predicting accurately the real energy content of the cosmos [24] [25]. Either way the rationale behind the dark dimensions and the quantum entanglement interpretation is the same. The infinitely many gaps in the fabric of space-time itself are what simulate quantum entanglement or hidden dark dimensions reduction of the total energy predicted by the classical formula by as much as 95.5\% [1] [2]. There are a few remarkable topological quantities involved in our present theory, the most important of which are the speed of light $\phi$, the intrinsic mass $\phi^{3}$ the five dimensional Kaluza-Klein mass 5 and the Hausdorff dimension of the empty set $\phi^{2}$ where $\phi=2 /(1+\sqrt{5})$. The present result confirms that the cosmological measurements are accurate and fully deserved the 2011 Nobel Prize and that the VSL theory of Magueijo and Smolin is a valid and accurate theory [9] [10]. Having come that far we must conclude that dark energy is not fiction but reality connected to the absolute value of the $95.5 \%$ negative energy of the quantum wave which is dual to the real $4.5 \%$ energy of the quantum particle. The same conclusion is obtained using pure energy considerations based on Einstein's field equation in the absence of matter field using the canonical equation of the 
degrees of freedom $D=d(d-3) / 2$. The only rational reason for the inability of our measurement instruments to register dark energy is the wave collapse on measurement. There seems to be at present one way to come around this problem to measure and harness dark energy and that is quantum non-demolition technology [21] [22]. We may also mention that dark energy seems to be, theoretically speaking, a natural result of the collapse of the Hawking-Hartle quantum wave of the cosmos on measurement which is inextricably and irreducibly linked to the vacuum state of Einstein's field equation. In this case two dimensional pure gravity would have a negative one degree of freedom resembling the empty set of the quantum wave behind dark energy while three dimensional pure gravity has zero degrees of freedom resembling the zero set and the quantum particle producing ordinary energy [16]. In conclusion we should mention that the Author's latest investigation hints strongly towards a strong link between Hooft-Veltman-Wilson type and 3.99 dimensional regularization space-time analysis and our $4-k$ fractal space-time dimension between the GUT and Planck energy scale where $k=\phi^{3}\left(1-\phi^{3}\right)=2 \phi^{5}$ which leads to our present exact dark energy density. This and other related results will be given in a forthcoming publication.

If we were asked to summarize the importance and deep meaning of the present work in a very few lines, we would say the following:

Remembering J. A. Wheeler's slogan, the boundary of a boundary is empty [28] as well as Hartle-Hawking no boundary proposal [29], we think that the present work clearly shows that the boundary of the boundary of the universe is the empty set which produces the $95.5 \%$ dark energy pushing the cosmos apart with negative gravity force while we are living locally inside the boundary surrounded by the ordinary $4.5 \%$ energy density and positive gravity. Thus the empty set is not only the beginning of everything in mathematics but also in physics [23] [30].

\section{References}

[1] Amendola, L. and Tsujikawa, S. (2010) Dark Energy: Theory and Observations. Cambridge University Press, Cambridge.

[2] Baryshev, Y. and Teerikorpi, P. (2002) Discovery of Cosmic Fractals. World Scientific, Singapore.

[3] Nottale, L. (2011) Scale Relativity. Imperial College Press, London.

[4] Ord, G. (1983) Fractal Space-Time. A geometric Analogue of Relativistic Quantum Mechanics. Journal of Physics A: Mathematical and General, 16, 1869. http://dx.doi.org/10.1088/0305-4470/16/9/012

[5] El Naschie, M.S. (2011) Quantum Entanglement as a Consequence of a Cantorian Micro Space-Time Geometry. Journal of Quantum Information Science, 1, 50-53. http://www.SCRIP.org/journal/jqis

[6] He, J.-H., et al. (2011) Quantum Golden Mean Entanglement Test as the Signature of the Fractality of Micro Space-Time. Nonlinear Science Letters B, 1, 45-50.

[7] El Naschie, M.S. (2009) The Theory of Cantorian Space-Time and High Energy Particle Physics (An Informal Review). Chaos, Solitons \& Fractals, 41, 2635-2646. http://dx.doi.org/10.1016/j.chaos.2008.09.059

[8] El Naschie, M.S. (2004) A Review of E-Infinity Theory and the Mass Spectrum of High Energy Particle Physics. Chaos, Solitons \& Fractals, 19, 209-236. http://dx.doi.org/10.1016/S0960-0779(03)00278-9

[9] Mageuijo, J. and Smolin, L. (2001) Lorentz Invariance with an Invariant Energy Scale. arXiv: hep-th/0112090V2.

[10] Mageuijo, J. (2003) Faster Than the Speed of Light. William Heinemann, London.

[11] El Naschie, M. S. (2006) On an Eleven Dimensional E-Infinity Fractal Space-Time Theory. International Journal of Nonlinear Sciences and Numerical Simulation, 7, 407-409.

[12] El Naschie, M. S. (2006) The "Discrete" Charm of Certain Eleven Dimensional Space-Time Theory. International Journal of Nonlinear Sciences and Numerical Simulation, 7, 477-481.

[13] Duff, M. (1999) The World in Eleven Dimensions. IOP Publishing Ltd., Bristol.

[14] Yau, S. T. and Nadis, S. (2010) The Shape of Inner Space. Basic Book, Persons Group, New York.

[15] Randal, L. (2005) Warped Passages. Allen Lane-Penguin Books, London.

[16] Penrose, R. (2004) The Road to Reality. Jonathan Cape, London.

[17] Becker, K., Becker, M. and Schwarz, J. (2007) String Theory and M-Theory. Cambridge University Press, Cambridge.

[18] Schwarz, P.M. and Schwarz, J.H. (2004) Special Relativity from Einstein to Strings. Cambridge University Press, Cambridge. http://dx.doi.org/10.1017/CBO9780511755811 
[19] Hardy, L. (1993) Non-Locality of Two Particles without Inequalities for Almost All Entangled States. Physical Review Letters, 71, 1665-1668. http://dx.doi.org/10.1103/PhysRevLett.71.1665

[20] Bengtsson, I. and Zyczkowski, K. (2008) Geometry of Quantum States. Cambridge University Press, Cambridge.

[21] Nakajima, S. and Murayama, Y. (Eds.) (1996) Foundations of Quantum Mechanics in the Light of New Technologies. World Scientific, Singapore.

[22] Braginsky, V. and Vyatchanin, S.P. (1981) Dokl. Akad. Nauk SSSR, 259, 570 [Sov. Phys. Dokl., 27, (1982), 478].

[23] He, J.H. and El Naschie, M.S. (2012) On the Monadic Nature of Quantum Gravity as Highly Structured Golden Ring Spaces and Spectra. Fractal Space-Time and Non-Commutative Geometry in Quantum and High Energy Physics, 3, 94-98, Asian Academic Publisher Limited, Hong Kong.

[24] El Naschie, M.S. (2013) A Resolution of Cosmic Dark Energy via a Quantum Entanglement Relativity Theory. Journal of Quantum Information Since, 3, 23-26.

[25] Marek-Crnjac, L., He, J.H. and El Naschie, M.S. (2013) Chaotic Fractals at the Root of Relativistic Quantum Physics and Cosmology. International Journal of Modern Nonlinear Theory and Application, 2, 78-88.

[26] El Naschie, M.S. (2013) A Rindler-KAM Space-Time Geometry and Scaling the Planck Scale Solves Quantum Relativity and Explains Dark Energy. International Journal of Astronomy and Astrophysics, 3, 483-493. http://dx.doi.org/10.4236/ijaa.2013.34056

[27] El Naschie, M.S. (2013) From Yang-Mills Photon in Curved Space-Time to Dark Energy Density. Journal of Quantum Information Science, 3, 121-126. http://dx.doi.org/10.4236/jqis.2013.34016

[28] Kheyfets, A. and Wheeler, J.A. (1986) Boundary of a Boundary Principle and Geometric Structure of Field Theories. International Journal of Theoretical Physics, 25, 573-580.

[29] Hartle, J.P. (1983) Wave Function of the Universe. Physical Review D, 28, 2960. http://dx.doi.org/10.1103/PhysRevD.28.2960

[30] Marek-Crnjac, L. (2013) Cantorian Space-Time Theory-The Physics of Empty Sets in Connection with Quantum Entanglement and Dark Energy. Lambert Academic Publishing, Saarbrücken. 
Scientific Research Publishing (SCIRP) is one of the largest Open Access journal publishers. It is currently publishing more than 200 open access, online, peer-reviewed journals covering a wide range of academic disciplines. SCIRP serves the worldwide academic communities and contributes to the progress and application of science with its publication.

Other selected journals from SCIRP are listed as below. Submit your manuscript to us via either submit@scirp.org or Online Submission Portal.
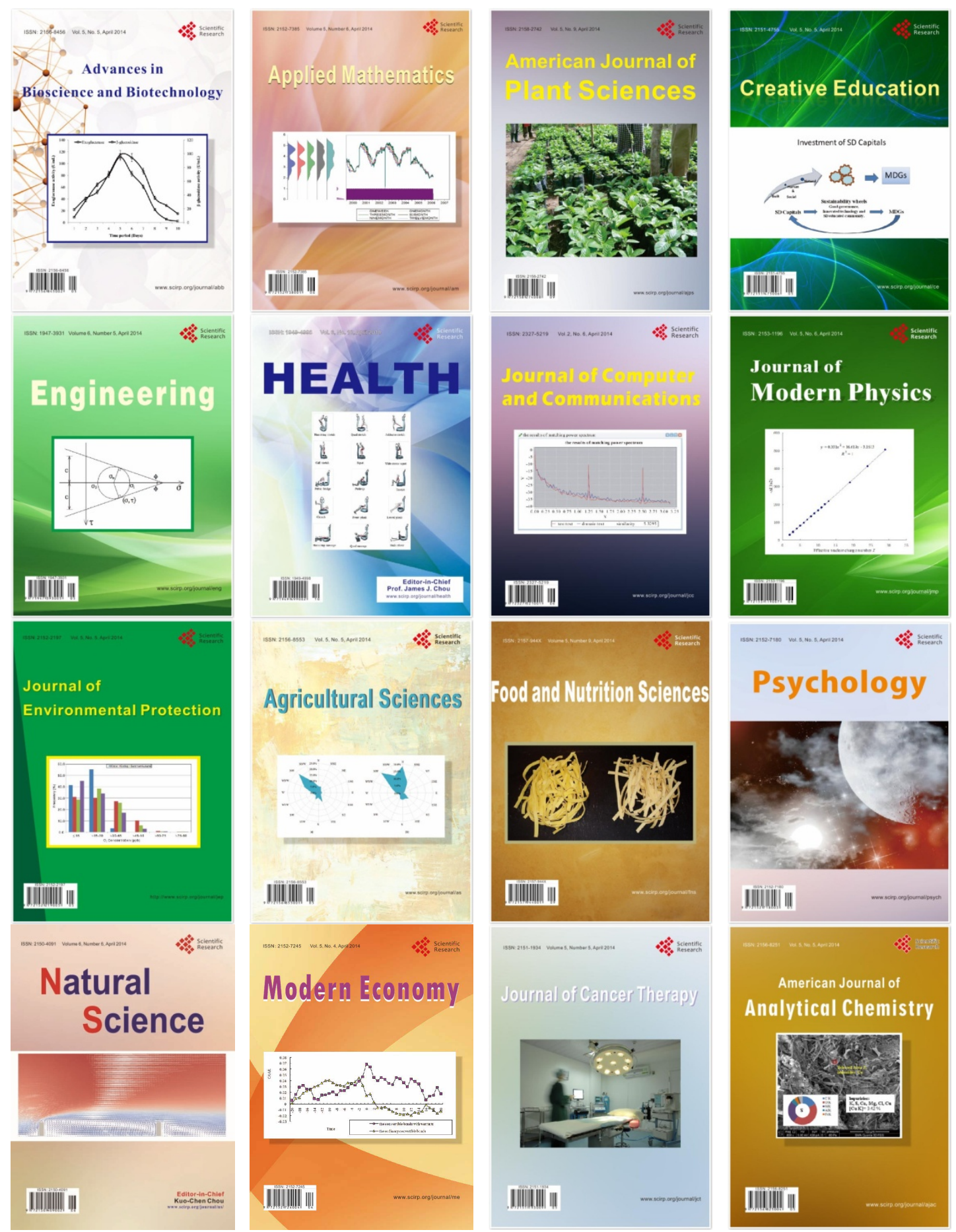